\title{
Predicting drug response of tumors from integrated genomic profiles by deep neural networks
}

Yu-Chiao Chiu', Hung-l Harry Chen ${ }^{1,2}$, Tinghe Zhang ${ }^{2}$, Songyao Zhang ${ }^{2,3}$, Aparna Gorthi', Li-Ju Wang ${ }^{1}$, Yufei Huang ${ }^{2,4^{*}}$ and Yidong Chen ${ }^{1,4^{*}}$

From The International Conference on Intelligent Biology and Medicine (ICIBM) 2018

Los Angeles, CA, USA. 10-12 June 2018

\begin{abstract}
Background: The study of high-throughput genomic profiles from a pharmacogenomics viewpoint has provided unprecedented insights into the oncogenic features modulating drug response. A recent study screened for the response of a thousand human cancer cell lines to a wide collection of anti-cancer drugs and illuminated the link between cellular genotypes and vulnerability. However, due to essential differences between cell lines and tumors, to date the translation into predicting drug response in tumors remains challenging. Recently, advances in deep learning have revolutionized bioinformatics and introduced new techniques to the integration of genomic data. Its application on pharmacogenomics may fill the gap between genomics and drug response and improve the prediction of drug response in tumors.

Results: We proposed a deep learning model to predict drug response (DeepDR) based on mutation and expression profiles of a cancer cell or a tumor. The model contains three deep neural networks (DNNs), i) a mutation encoder pre-trained using a large pan-cancer dataset (The Cancer Genome Atlas; TCGA) to abstract core representations of high-dimension mutation data, ii) a pre-trained expression encoder, and iii) a drug response predictor network integrating the first two subnetworks. Given a pair of mutation and expression profiles, the model predicts $\mathrm{IC}_{50}$ values of 265 drugs. We trained and tested the model on a dataset of 622 cancer cell lines and achieved an overall prediction performance of mean squared error at 1.96 (log-scale $I C_{50}$ values). The performance was superior in prediction error or stability than two classical methods (linear regression and support vector machine) and four analog DNN models of DeepDR, including DNNs built without TCGA pre-training, partly replaced by principal components, and built on individual types of input data. We then applied the model to predict drug response of 9059 tumors of 33 cancer types. Using per-cancer and pan-cancer settings, the model predicted both known, including EGFR inhibitors in non-small cell lung cancer and tamoxifen in ER+ breast cancer, and novel drug targets, such as vinorelbine for TTN-mutated tumors. The comprehensive analysis further revealed the molecular mechanisms underlying the resistance to a chemotherapeutic drug docetaxel in a pan-cancer setting and the anti-cancer potential of a novel agent, CX-5461, in treating gliomas and hematopoietic malignancies.

(Continued on next page)
\end{abstract}

\footnotetext{
* Correspondence: Yufei.Huang@utsa.edu; ChenY8@uthscsa.edu

${ }^{2}$ Department of Electrical and Computer Engineering, The University of Texas

at San Antonio, San Antonio, TX 78249, USA

'Greehey Children's Cancer Research Institute, University of Texas Health

Science Center at San Antonio, San Antonio, TX 78229, USA

Full list of author information is available at the end of the article
}

(c) The Author(s). 2019 Open Access This article is distributed under the terms of the Creative Commons Attribution 4.0 International License (http://creativecommons.org/licenses/by/4.0/), which permits unrestricted use, distribution, and reproduction in any medium, provided you give appropriate credit to the original author(s) and the source, provide a link to the Creative Commons license, and indicate if changes were made. The Creative Commons Public Domain Dedication waiver (http://creativecommons.org/publicdomain/zero/1.0/) applies to the data made available in this article, unless otherwise stated. 
(Continued from previous page)

Conclusions: Here we present, as far as we know, the first DNN model to translate pharmacogenomics features identified from in vitro drug screening to predict the response of tumors. The results covered both well-studied and novel mechanisms of drug resistance and drug targets. Our model and findings improve the prediction of drug response and the identification of novel therapeutic options.

Keywords: Deep neural networks, Pharmacogenomics, Drug response prediction, Cancer cell line encyclopedia, Genomics of Drug Sensitivity in Cancer, The Cancer Genome Atlas

\section{Background}

Due to tumor heterogeneity and intra-tumor sub-clones, an accurate prediction of drug response and an identification of novel anti-cancer drugs remain challenging tasks [1, 2]. Pharmacogenomics, an emerging field studying how genomic alterations and transcriptomic programming determine drug response, represents a potential solution [3, 4]. For instance, recent reports identified mutation profiles associated with drug response both in tumor type-specific and pan-cancer manners [5, 6]. As drug response data of large patient cohorts are scarcely available, large-scale cell line-based screening can greatly facilitate the study of pharmacogenomics in cancer. Recently, the Genomics of Drug Sensitivity in Cancer (GDSC) Project proposed a comprehensively landscape of drug response of $\sim 1000$ human cancer cell lines to 265 anti-cancer drugs and unveiled crucial oncogenic aberrations related to drug sensitivity $[7,8]$. Because of the fundamental differences between in vitro and in vivo biological systems, a translation of pharmacogenomics features derived from cells to the prediction of drug response of tumors is to our knowledge not yet realized.

Deep learning (DL) is the state-of-the-art machine learning technology for learning knowledge from complex data and making accurate predictions. It features the ability to learn the representation of data without the need for prior knowledge and an assumption on data distributions. The DL technology has been successfully applied to bioinformatics studies of regulatory genomics, such as predicting binding motifs [9], investigating DNA variants [10], deciphering single-cell omics $[11,12]$, and extraction of genomics features for survival prediction [13]. In pharmaceutical and pharmacogenomics research, reports have shown its ability to predict drug-target interactions [14], screen for novel anti-cancer drugs [15], and predict drug synergy [16]. Nevertheless, data complexity and the requirement of large training datasets have limited its application to integrate genomics data and comprehensively predict drug response, hindering the translation to precision oncology.

Addressing the unmet demands, the present study is aimed to predict the response of tumors to anti-cancer drugs based on genomic profiles. We designed DeepDR, a deep neural network (DNN) model to learn the genetic background from high-dimensional mutation and expression profiles using the huge collection of tumors of The Cancer Genome Atlas (TCGA). The model was further trained by the pharmacogenomics data developed in human cancer cell lines by the GDSC Project and their corresponding genomic and transcriptomic alterations, and finally applied to TCGA data again to predict drug response of tumors. Collectively, DeepDR is a novel DL model that translates cell line-derived pharmacogenomics knowledge via tumor genomic and transcriptomic abstraction to predict tumors' response to compound treatment.

\section{Methods \\ Datasets}

We downloaded gene-level expression data of 935 cell lines of the Cancer Cell Line Encyclopedia (CCLE) and 11,078 TCGA pan-cancer tumors from the $\mathrm{CTD}^{2}$ Data Portal [17] and UCSC TumorMap [18], respectively. Given the total numbers of cell lines, tumors, and genes as $C, T, G$, respectively, we metricized the expression data by $E^{C C L E}=\left\{\log _{2}\left(t p m_{g, c}^{C C L E}+1\right)\right\}$, where $t p m_{g, c}^{C C L E}$ is the number of transcripts per million of gene $g(g \in[1, G])$ in cell line $c(c \in[1, C])$, and $\boldsymbol{E}^{T C G A}=\left\{\log _{2}\left(t p m_{g, t}^{T C G A}+1\right)\right\}$, where $t p m_{g, t}^{T C G A}$ denotes the number of transcripts per million of the same gene in tumor $t(t \in[1, T])$. Genes with low information burden (mean $<1$ or st. dev. $<0.5$ ) among TCGA samples were removed. Mutation Annotation Format (MAF) files of mutation data were downloaded directly from CCLE (1463 cells) [19, 20] and TCGA databases (10,166 tumors). Here we only considered four types of nonsynonymous mutations, including missense and nonsense mutations, and frameshift insertions and deletions. Thus, we had binary matrices of $M^{C C L E}=\left\{m_{g, c}^{C C L E}\right\}$ and $\boldsymbol{M}^{T C G A}=\left\{m_{g, t}^{T C G A}\right\}$, where $m_{g, c}^{C C L E}$ and $m_{g, t}^{T C G A}$ are the mutation states ( 1 for mutation and 0 for wildtype) of gene $g$ in $c$ and $t$, respectively. Genes with no mutations in CCLE and TCGA samples were eliminated. 
We also downloaded drug response data of 990 CCLE cell lines to 265 anti-cancer drugs measured by the half maximal inhibitory concentration $\left(\mathrm{IC}_{50}\right)$ from the GDSC Project [7]. $\mathrm{IC}_{50}$ were measured in $\mu \mathrm{M}$ and represented in $\log$ scale (i.e., $I C^{C C L E}=\left\{\log _{10}\left(i c_{d, c}^{C C L E}\right)\right\}$, with $d$ denoting the $d$-th drug and $d \in[1, D])$ and missing data were imputed by a weighted mean of $\mathrm{IC}_{50}$ of 5 nearest drugs using $\mathrm{R}$ packages VIM and laeken [21, 22]. In this study, we analyzed 622 cell lines with available expression, mutation, and $\mathrm{IC}_{50}$ data and 9059 tumors with expression and mutation profiles.

\section{General settings of DNNs and computation environment} DNN training in this study were performed using the python library Keras 1.2.2 with TensorFlow backend. We used fully (or densely) connected layers for all networks. At a neuron $j$, its output $y_{j}$ is calculated by

$$
y_{j}=F\left(\sum_{i} w_{i j} x_{i}+b_{j}\right)
$$

, where $x_{i}$ is the output of neuron $i$ at the previous layer of $j, w_{i j}$ and $b_{j}$ denote the synaptic weight and bias, respectively, and $F$ represents an activation function. The notation of all neurons at a layer can thus be written as

$$
\boldsymbol{y}=F(\boldsymbol{w} \boldsymbol{x}+\boldsymbol{b}) .
$$

During training, synaptic weights and biases are adjusted to minimize a loss function. We hereafter refer to the two parameters as synaptic parameters because they represent the model and can be used to transfer a learned model to another. In this study, DNNs were optimized using the Adam optimizer with a loss function of mean squared error (MSE). We used the He's uniform distribution [23] to initialize autoencoders and the Prediction $(\mathrm{P})$ network, while the mutation encoder $\left(\mathrm{M}_{\mathrm{enc}}\right)$ and expression encoder $\left(\mathrm{E}_{\mathrm{enc}}\right)$ in the complete model were initialized by the synaptic parameters learned from the pre-training on TCGA data. Neuron activation function was set as rectified linear unit (ReLU) except for the output layer of $\mathrm{P}$ as linear in order to better fit the distribution of log-scale $\mathrm{IC}_{50}$.

\section{Overview of DeepDR}

DeepDR was developed to predict $\mathrm{IC}_{50}$ values based on genomic profiles of a cell or a tumor. Given the pair of mutation and expression vectors of sample $c,\left\{M^{C C L E}(:, c)\right.$, $\left.\boldsymbol{E}^{C C L E}(:, c)\right\}$, the model predicts a $D$-length vector of $\mathrm{IC}_{50}$, $I^{C C C L E}(c)$, as an output. As shown in Fig. 1, the model is composed of three networks: i) a mutation encoder $\left(M_{\text {enc }}\right)$, ii) an expression encoder $\left(E_{e n c}\right)$, and iii) a prediction feedforward network (P). The first and second components are the encoding parts of two autoencoders pre-trained using TCGA data to transform high-order features of mutation and expression data into a lower dimensional representation. The encoded representations of mutation and expression profiles were linked into $\mathrm{P}$ and the entire model was trained on CCLE data to make prediction of $\mathrm{IC}_{50}$ values. Details of DeepDR are described below.

\section{Pre-training of mutation and expression encoders}

Autoencoder is an unsupervised DL architecture that includes a symmetric pair of encoder and decoder. By minimizing the loss between input and reconstructed (i.e., decoded) data, it reduces the dimension of complex data and captures crucial features at the bottleneck layer (the layer between encoder and decoder) (Fig. 1b, top and bottom panels). We pre-trained an autoencoder on each of the TCGA mutation and expression datasets to optimize the capability to capture high-order features. To determine the optimized architecture, we adopted a hyper-parameter optimization method, namely hyperas [24], to select i) number of neurons at the 1st layer (4096, 2048, or 1024), ii) number of neurons at the 2nd layer $(512,256$, or 128), iii) number of neurons at the 3rd layer (the bottleneck layer; 64, 32, or 16), and iv) batch size (128 or 64). Each combination was trained for 20 epochs; the best-performing model was re-run for 100 epochs and the synaptic parameters were saved.

\section{Complete prediction network}

In our complete model, encoders of the two optimized autoencoders, i.e., $\mathrm{M}_{\text {enc }}$ and $\mathrm{E}_{\mathrm{enc}}$, were linked to $\mathrm{P}$ to make predictions of $\mathrm{IC}_{50}$ (Fig. 1). $\mathrm{P}$ is a 5-layer feedforward neural network, including the first layer merging output neurons of the two encoders, three fully connected layers, and the last layer of $D$ neurons generating $\mathrm{IC}_{50}$ values of $D$ drugs (Fig. $1 \mathrm{~b}$, orange box). In the complete model, architecture (number of layers and number of neurons at each layer) of $M_{\text {enc }}$ and $E_{\text {enc }}$ was fixed; their synaptic parameters were initialized using the parameters obtained from pre-training in TCGA and updated during the training process. $\mathrm{P}$ was randomly initialized. We trained the entire model using CCLE data, with 80,10 , and $10 \%$ of samples as training, validation, and testing sets, respectively. We note the validation dataset was not used to update model parameters but to stop the training process when the loss in validation set had stopped decreasing for 3 consecutive epochs to avoid model overfitting. Performance of the model was evaluated using the testing samples, i.e., $\operatorname{MSE}\left(\widehat{I C C C L E}\left(:, C_{\text {test }}\right)\right.$, $\left.\operatorname{IC}^{C C L E}\left(:, C_{\text {test }}\right)\right)$, where $C_{\text {test }}$ denotes the test set of cell lines.

We applied the final model to predict drug response of TCGA tumors. For a tumor $t,\left\{\boldsymbol{M}^{T C G A}(:, t), \boldsymbol{E}^{T C G A}(:, t)\right\}$ 

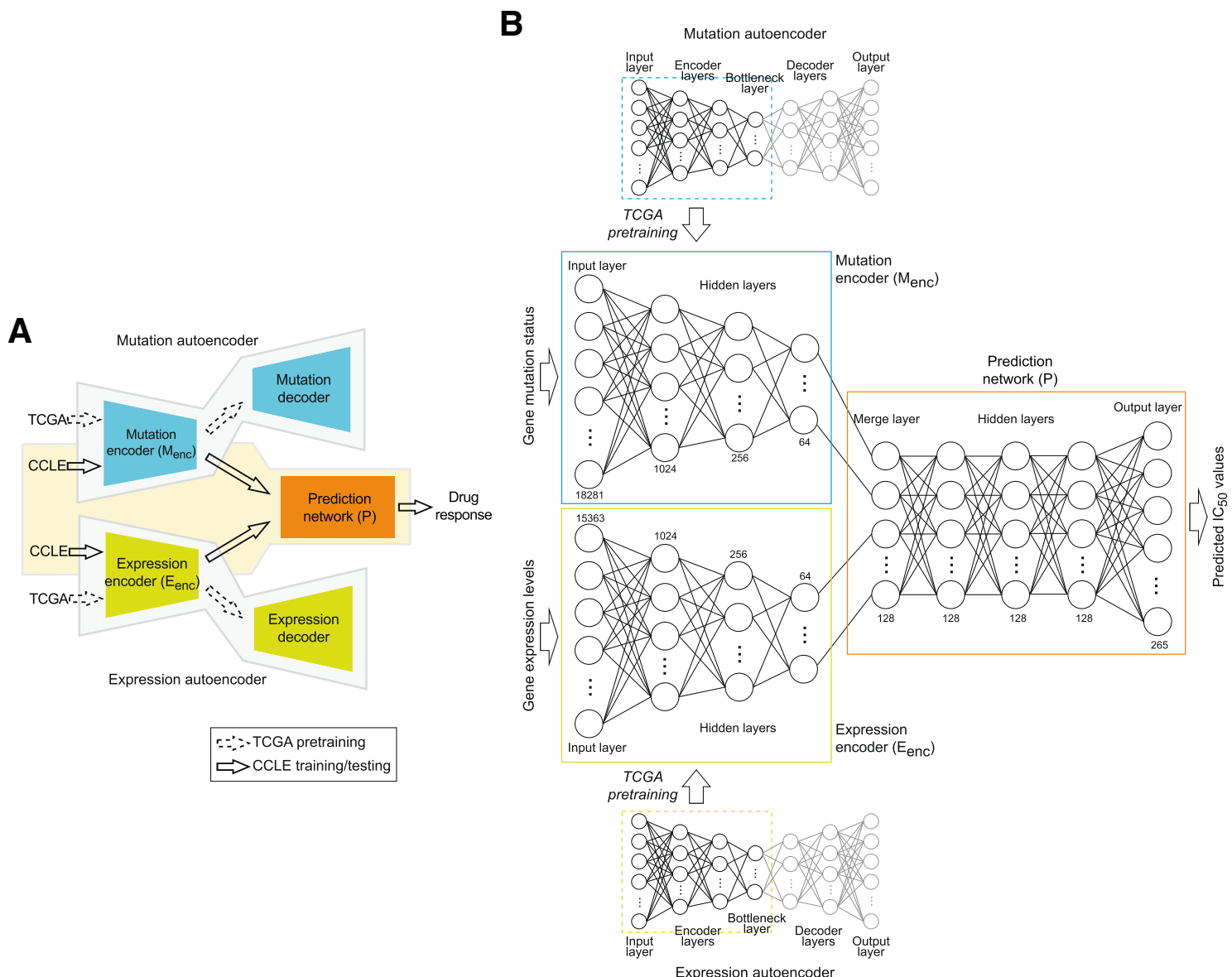

Fig. 1 Illustration of DeepDR. (a) Model overview. Mutation and expression data of TCGA ( $n=9059)$ were used to pre-train two autoencoders (highlighted in blue and green) to extract data representations. Encoders of the autoencoders, namely mutation encoder $\mathrm{M}_{\text {enc }}$ and expression encoder $E_{e n c}$, were linked to a prediction network ( $P$; denoted in orange) and the entire network (i.e., $M_{\text {enc, }} E_{e n c}$ and $P$ ) was trained using CCLE data ( $n=622$, of which 80,10 , and 10\% used as training, validation, and testing, respectively) to predict the response to 265 drugs. (b) Architecture of the neural networks. Numbers denote the number of neurons at each layer

was fed into the model and $\widehat{I C T C A}(:, t)$ was calculated. A high predicted $\mathrm{IC}_{50}$ indicates an adverse response of a patient to the corresponding drug.

\section{Comparison to other model designs}

Performance of DeepDR was compared to four different DNN designs. First, to assess the effect of TCGA pre-training on $M_{\text {enc }}$ and $E_{\text {enc }}$, we randomly initialized both encoders using the He's uniform distribution and calculated MSE of the entire model. Second, dimension reduction of the $M_{\text {enc }}$ and $E_{\text {enc }}$ networks was replaced by principal component analysis (PCA). Last two models were built without $M_{e n c}$ or $E_{\text {enc }}$ to study whether they jointly improved the performance. In each iteration, CCLE samples were randomly assigned to training (80\%), validation (10\%), and testing (10\%) and each model was trained and tested. Performance in terms of the number of consumed epochs and MSE in $\mathrm{IC}_{50}$ were summarized and compared across the 100 iterations. We also analyzed two classical prediction methods, multivariate linear regression and regularized support vector machine (SVM). For each method, top 64 principal components of mutations and gene expression were merged to predict $\mathrm{IC}_{50}$ values of all (using linear regression) or individual drugs (SVM).

\section{Results}

\section{Construction and evaluation of DeepDR in CCLE}

The study is aimed to predict drug response (measured as log-scale $\mathrm{IC}_{50}$ values) using genome-wide mutation and expression profiles. We included mutation and expression profiles of 622 CCLE cell lines of 25 tissue types and 9059 TCGA tumors of 33 cancer types. After data preprocessing, 18,281 and 15,363 genes with mutation and expression data, respectively, available in both CCLE and TCGA samples were analyzed. Log-scale $\mathrm{IC}_{50}$ values of all cell lines in response to 265 anti-cancer 
drugs were collected from the GDSC Project [7]. After imputation of missing values, the range of $\log \mathrm{IC}_{50}$ was from -9.8 to 12.8 with a standard deviation of 2.6 (Fig. 2a). We designed DeepDR with three building blocks: 4-layer $M_{e n c}$ and 4-layer $E_{e n c}$ for capturing high-order features and reducing dimensions of mutation and expression data, and a 5-layer prediction network $\mathrm{P}$ integrating the mutational and transcriptomic features to predict $\mathrm{IC}_{50}$ of multiple drugs (Fig. 1). To make the best use of the large collection of TCGA pan-cancer data, we pre-trained an autoencoder for each data type and extracted the encoders, $\mathrm{M}_{\mathrm{enc}}$ (number of neurons at each layer, 18,281, 1024, 256, and 64) and $E_{\text {enc }}(15,363,1024,256$, and 64$)$, to construct our final model (detailed in Methods). Output neurons of the two encoders were linked to $\mathrm{P}$ (number of neurons at each layer, $64+64,128,128,128$, and 265), of which the last layer outputs predicted $\mathrm{IC}_{50}$. Architecture of the complete neural networks is shown in Fig. $1 \mathrm{~b}$.

After pre-training $M_{\text {enc }}$ and $E_{\text {enc }}$ components, we trained the entire model using $80 \%$ of CCLE samples together with a validation set of $10 \%$ of samples to avoid overfitting. The remaining samples (64 cells; 16,960 cell-drug combinations) were used for testing. The model achieved an overall MSE in $\mathrm{IC}_{50}$ of 1.53 , corresponding to 1.48 and 1.98 in training/validation and testing data, respectively. Generally, the distribution of predicted $\mathrm{IC}_{50}$ was similar to original data (Fig. 2a-b), while the two modes of original data seemed to be enhanced (highlighted in Fig. 2a). In both training/validation and testing data, the prediction was highly consistent to the true data in terms of $\mathrm{IC}_{50}$ values (Pearson correlation; $\rho_{P}$ ) and rank of drugs (Spearman correlation; $\left.\rho_{S}\right)$ of a sample $\left(\rho_{P} \in[0.70,0.96], \rho_{S} \in[0.62,0.95]\right.$, and all $P$-values $<1.0 \times 10^{-29}$; Fig. $2 \mathrm{c}-\mathrm{d}$ ). Of note, correlations achieved in training/validation and testing samples were highly comparable (Fig. 2c-d), confirming the performance of our model.

\section{Performance comparisons to other designs}

To test the stability of DeepDR, we ran 100 training processes each of which training, validation, and testing cells were reselected. Overall, the model converged in 14.0 epochs (st. dev., 3.5; Table 1) and achieved an MSE of 1.96 in testing samples (st. dev., 0.13; Fig. 2e and Table 1). We compared the performance to linear regression, SVM, and four analog DNNs of DeepDR, including random initialization (identical architecture, but without TCGA pre-training of $\mathrm{M}_{\mathrm{enc}}$ and $\mathrm{E}_{\text {enc }}$ ), PCA $\left(M_{\text {enc }}\right.$ and $E_{\text {enc }}$ each replaced by top 64 principal

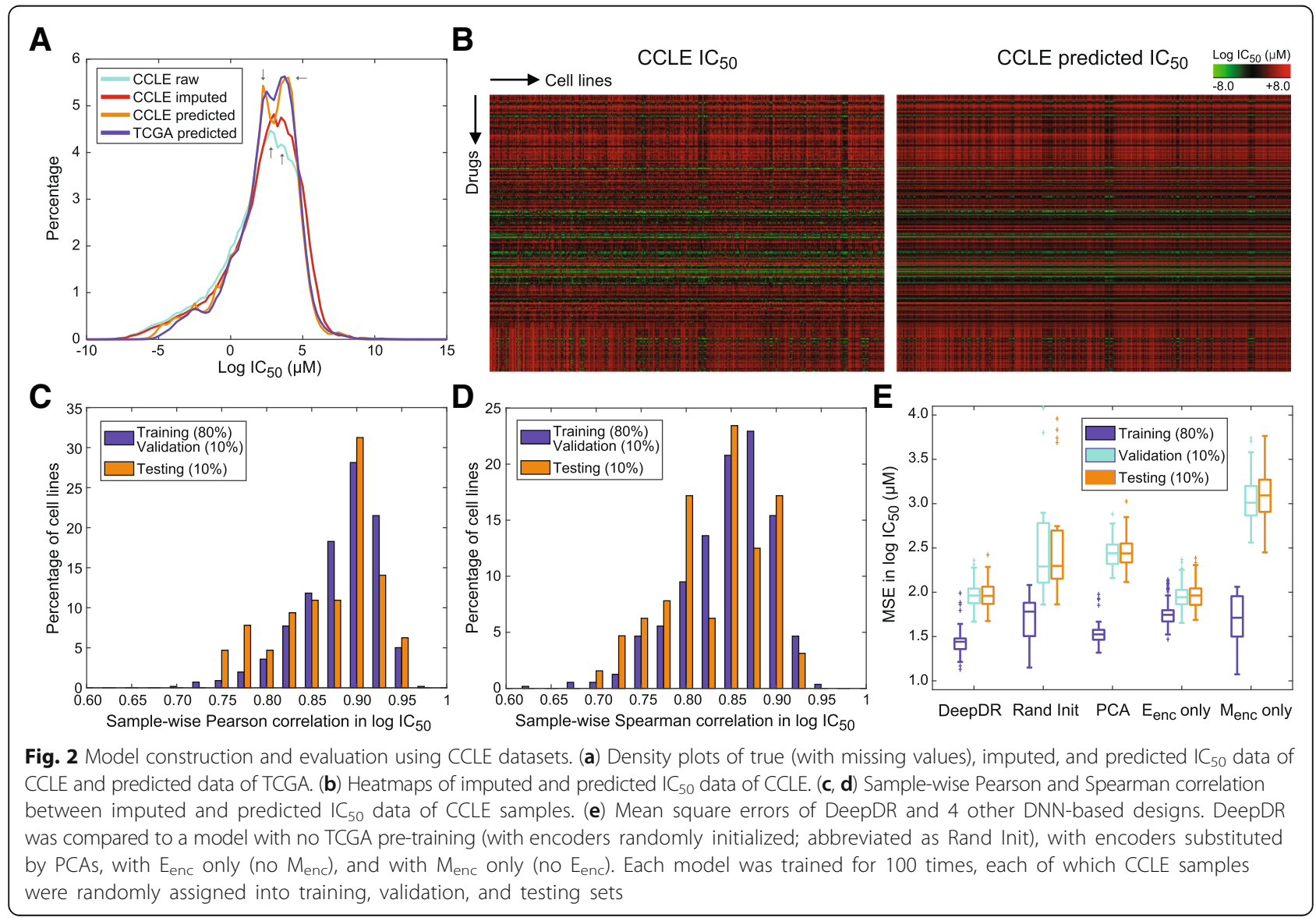


Table 1 Performance of DeepDR and other models

\begin{tabular}{llllllll}
\hline Measurement & DeepDR & Linear regression & SVM & Random initialization & PCA & $E_{\text {enc }}$ only & $M_{\text {enc }}$ only \\
\hline Median MSE in testing samples $^{\mathrm{a}}$ & 1.96 & $10.24^{\mathrm{b}}$ & $8.92^{\mathrm{c}}$ & 2.30 & 2.44 & 1.96 & 3.09 \\
Median number of training epochs $^{\mathrm{a}}$ & 14 & - & - & 9 & 29 & 17 & 9.5
\end{tabular}

${ }^{a}$ Median of 100 shuffles of training, validation, and testing samples

${ }^{\mathrm{b}}$ Result of one multivariate regression model

${ }^{c}$ Results of 265 SVM models, each predicting $I C_{50}$ for a drug

components of mutation and expression data), $M_{\text {enc }}$ only $\left(E_{\text {enc }}\right.$ removed from DeepDR), and $E_{\text {enc }}$ only $\left(\mathrm{M}_{\mathrm{enc}}\right.$ removed from DeepDR). The two classical methods seemed to suffer from high MSE in testing samples (10.24 and 8.92 for linear regression and SVM, respectively; Table 1). DeepDR also outperformed DNNs with random initialization and PCA in MSE (difference in medians, 0.34 and 0.48; Fig. 2e and Table 1) and stability (st. dev. of MSE in testing samples $=0.13,1.21$, and 0.17 for DeepDR, random initialization, and PCA, respectively; Fig. 2e). While the $\mathrm{E}_{\text {enc }}$-only model achieved similar performance to our model (difference in medians $=0.0042$; Fig. 2E and Table 1), the addition of $M_{\text {enc }}$ seemed to bring faster convergence (difference in medians $=3$; Table 1). Our data echoed the biological premise that gene expressions are more directly linked to biological functions and thus richer in information burden than mutations.

\section{Associations of gene mutations to predicted drug response in TCGA - Per-cancer study}

In search of effective anti-cancer drugs in tumors, we applied DeepDR directly to predict the response of 9059 TCGA samples to the 265 anti-cancer drugs. The predicted $\mathrm{IC}_{50}$ values followed a similar distribution to CCLE cells (Fig. 2a, blue line). Realizing the different nature of cell lines and tumors, we started by examining several drugs with well-known target genes. As shown in Fig. 3a, breast invasive carcinoma (BRCA) with positive estrogen receptor (ER; assessed by immunohistochemistry by TCGA) responded to a selective estrogen receptor modulator, tamoxifen, significantly better than ER-negative patients $\left(t\right.$-test $\left.P=2.3 \times 10^{-4}\right)$. Also, two EGFR inhibitors, afatinib and gefitinib, achieved better performance in non-small cell lung cancers (NSCLC) with mutated EGFR $\left(P=2.0 \times 10^{-7}\right.$ and $\left.6.6 \times 10^{-3}\right)$. While the promising results on these well-characterized drugs showed the applicability of our model to tumors,

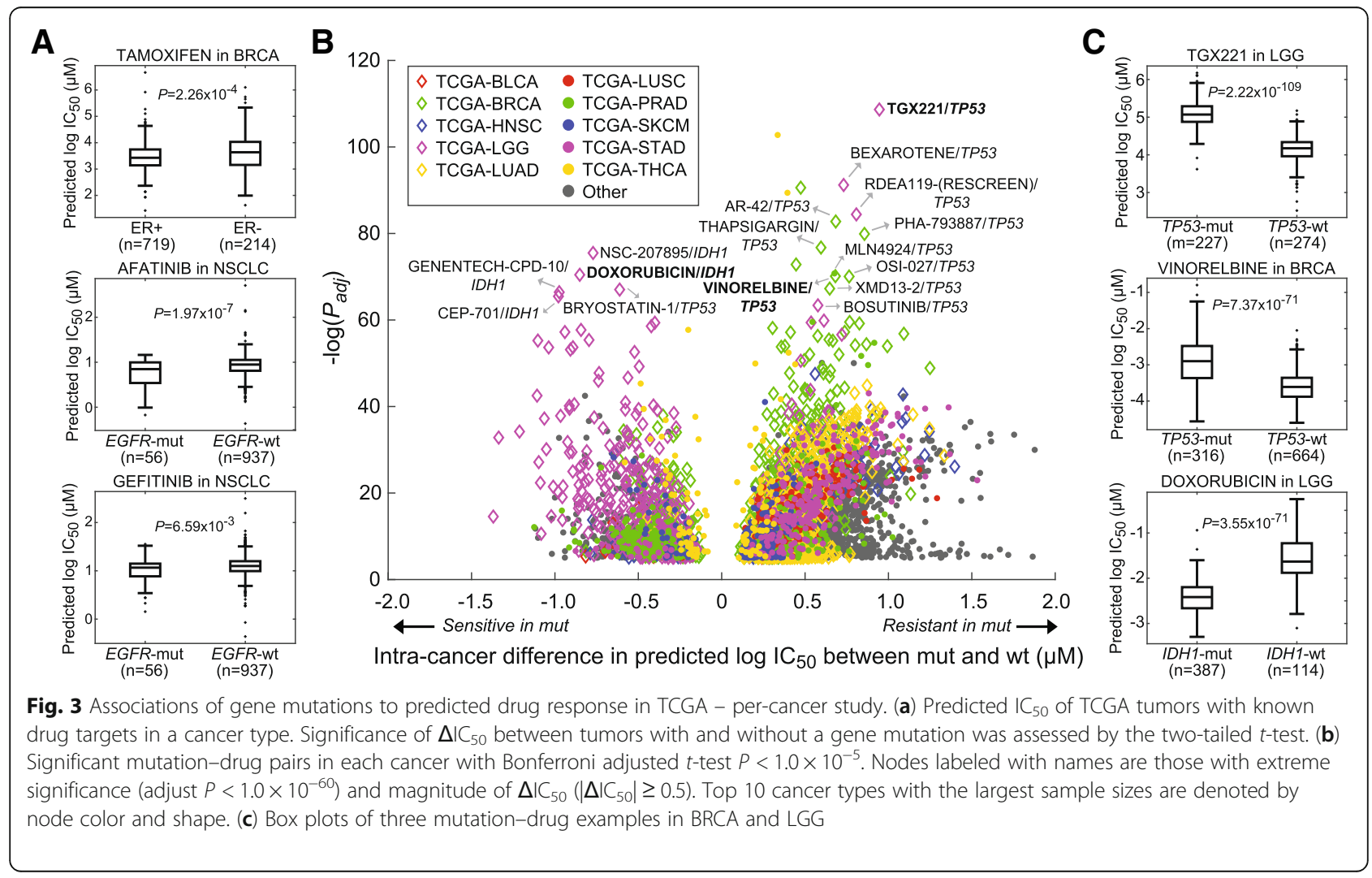


we noted that the magnitude of differences in predicted $\mathrm{IC}_{50}$ levels was modest, underlining the fundamental differences between cell lines and tumors. In order to prioritize mutations underlying drug response, we systematically analyzed all cancer-mutation-drug combinations and tested the significance of differences in $\mathrm{IC}_{50}$ between samples with and without a mutation for each cancer. Here only genes with a mutation rate higher than $10 \%$ and harbored by at least 10 patients in a cancer were analyzed. With a stringent criterion of Bonferroni-adjusted $t$-test $P<1.0 \times 10^{-5}$, we identified a total of 4453 significant cancer-mutation-drug combinations involving 256 drugs and 169 cancer-mutation combinations (Fig. 3b). The top three combinations were TP53 mutations in lung adenocarcinoma (LUAD; modulating response to 235 drugs), lung squamous cell carcinoma (LUSC; 228 drugs), and stomach adenocarcinoma (STAD; 224 drugs) (Table 2). TP53 was one of the most frequently mutated and well-studied genes in many cancers. The mutation has been shown to be associated with cancer stem cells and resistance functions and thus regulates drug resistance $[25,26]$. For instance, our data indicated its associations with resistance of a PI3K $\beta$ inhibitor, TGX221, in 9 cancers including low-grade glioma (LGG; mean difference in $\mathrm{IC}_{50}$ $\left(\Delta \mathrm{IC}_{50}\right)=0.95 ; P=2.2 \times 10^{-109}$; Fig. 3c) and resistance of vinorelbine in BRCA $\left(\Delta \mathrm{IC}_{50}=0.68 ; P=7.4 \times 10^{-71}\right.$; Fig. $\left.3 \mathrm{c}\right)$ and 6 other cancers. We also identified gene mutations that sensitized tumors to a large number of drugs, such as $I D H 1$ (138 drugs; Table 2). IDH1 was the most frequently mutated gene in LGG (77.3\% in our data; Table 2) and known to regulate cell cycle of glioma cells and enhance the response to chemotherapy [27]. Our finding agreed with the report and showed that $I D H 1$ mutation dramatically reduced $\mathrm{IC}_{50}$ of chemotherapeutic agents, e.g., doxorubicin in LGG $\left(\Delta \mathrm{IC}_{50}=-0.85 ; P=3.6 \times 10^{-71} ;\right.$ Fig. $\left.3 \mathrm{c}\right)$.

\section{Associations of gene mutations to predicted drug response in TCGA - Pan-cancer study}

We also carried out a study to explore how gene mutations affect drug response in a pan-cancer setting. The analysis was focused on 11 genes with mutation rates higher than 10\% across all TCGA samples (Table 3). Using an identical criterion, we identified 2119 significant mutation-drug pairs composed of 256 drugs, among which 1882 (88.8\%) and 237 (11.2\%) were more resistant and sensitive in mutated samples, respectively (Fig. 4a and Table 3). TP53 (251 drugs), CSMD3 (223), SYNE1 (218), TTN (206), and RYR2 (199) were the top drug response-modulating genes (Table 3). Among them, TP53 (9 sensitive and 242 resistant drugs) and TTN mutations (44 and 162) were associated with the largest numbers of resistant and sensitive drugs, respectively (Table 3 ). Thus, we further investigated drugs associated with the 2 genes. Many of the drugs with large TP53 mutations-modulated changes in $\Delta \mathrm{IC}_{50}\left(\left|\Delta \mathrm{I} C_{50}\right| \geq 0.7\right.$; Fig. 4a-b) were previously studied in different cancer types by in vitro models. For instance, wildtype TP53 is required in the anti-cancer actions of CX-5461 $[28,29]$ and sorafenib [30] (both $P$ of $\Delta \mathrm{IC}_{50} \sim 0$ in our data; Fig. $\left.4 \mathrm{~b}\right)$, sensitizes various cancer cells to bortezomib [31] $\left(P=4.4 \times 10^{-308}\right.$; Fig. $\left.4 \mathrm{~b}\right)$, and enhances phenformin-induced growth inhibition and apoptosis [32] $\left(P=2.0 \times 10^{-241}\right.$; Fig. $\left.4 \mathrm{~b}\right)$. As for previously less explored TTN mutations, the longest gene in human genome known to carry a large number of variations, our data indicated that perhaps TTN acts as a marker gene of tumors sensitized to chemotherapeutic agents such as vinorelbine $(P \sim 0$; Fig. $4 C)$ and a potential anti-cancer drug epothilone $\mathrm{B}(P=$ $2.5 \times 10^{-253}$; Fig. 4c). Taken together findings from our per- and pan-cancer studies, we have demonstrated the applicability of our model to predict drug response of tumors and unveil novel and well-studied genes modulating drug response in cancer.

\section{Pharmacogenomics analysis of docetaxel and CX-5461 in TCGA}

To unveil the pharmacogenomics landscape of drugs, a comprehensive study of mutation and expression profiles associated with resistance of a drug in a pan-cancer

Table 2 Top mutations in modulating drug response among individual cancers

\begin{tabular}{|c|c|c|c|c|c|}
\hline Cancer & Gene & Mutation rate & Num. modulated drugs & Num. sensitive drugs & Num. resistant drugs \\
\hline LUAD & TP53 & $46.1 \%$ & 235 & 0 & 235 \\
\hline LUSC & TP53 & $75.1 \%$ & 228 & 0 & 228 \\
\hline STAD & TP53 & $43.3 \%$ & 224 & 0 & 224 \\
\hline HNSC & TP53 & $66.1 \%$ & 207 & 0 & 207 \\
\hline COAD & TP53 & $55.7 \%$ & 197 & 0 & 197 \\
\hline LIHC & TP53 & $27.0 \%$ & 194 & 1 & 193 \\
\hline BRCA & TP53 & $32.2 \%$ & 182 & 7 & 175 \\
\hline LGG & IDHI & $77.3 \%$ & 159 & 138 & 21 \\
\hline PRAD & TP53 & $10.8 \%$ & 146 & 1 & 145 \\
\hline KIRC & PBRM1 & $38.0 \%$ & 142 & 3 & 139 \\
\hline
\end{tabular}


Table 3 Top gene mutations modulating pan-cancer drug response

\begin{tabular}{lllll}
\hline Gene & Mutation rate & Num. modulated drugs & Num. sensitive drugs & Num. resistant drugs \\
\hline TP53 & $34.3 \%$ & 251 & 9 & 242 \\
CSMD3 & $12.6 \%$ & 223 & 12 & 211 \\
SYNE1 & $11.5 \%$ & 218 & 10 & 208 \\
TTN & $30.2 \%$ & 206 & 44 & 162 \\
RYR2 & $11.9 \%$ & 199 & 14 & 185 \\
USH2A & $10.7 \%$ & 191 & 12 & 179 \\
LRP1B & $12.1 \%$ & 188 & 19 & 169 \\
FLG & $11.0 \%$ & 183 & 9 & 174 \\
MUC16 & $19.5 \%$ & 161 & 51 & 110 \\
PCLO & $10.5 \%$ & 155 & 12 & 143 \\
PIK3CA & $11.7 \%$ & 144 & 45 & 99
\end{tabular}

setting was carried out. Here we took two drugs as demonstrating examples, a widely used chemotherapeutic agent docetaxel and a novel anti-cancer drug CX-5461 currently under investigation in several cancers. For each drug, pan-cancer patients predicted to be very sensitive and resistant (with $\mathrm{IC}_{50}$ in bottom and top $1 \%, n=91$ in each group; Fig. 5a, left panel) were compared for cancer type compositions, mutation rates, and differential gene expression. Top cancer types of docetaxel-sensitive patients were esophageal carcinoma (ESCA; 25.3\%), cervical and endocervical cancer (CESC; 13.2\%), and head and neck squamous cell carcinoma (HNSC; 9.9\%)
(Fig. 5b, left panel), while top resistant patients were mainly liver hepatocellular carcinoma (LIHC; 42.9\%), LGG (26.4\%), and glioblastoma multiforme (GBM; 12.1\%) (Fig. 5b, left panel). Top 10 gene with most changed mutation rates between the two groups of patients are listed in Fig. 5c. On average, each sensitive tumor harbored 2.7 mutations among these genes, much higher than 0.51 observed in the resistant group (Fig. 5c, left panel), implying tumors with higher mutation burdens in crucial genes may be more vulnerable to the treatment. Of note, a great majority of the most significantly differentially expressed genes were upregulated in
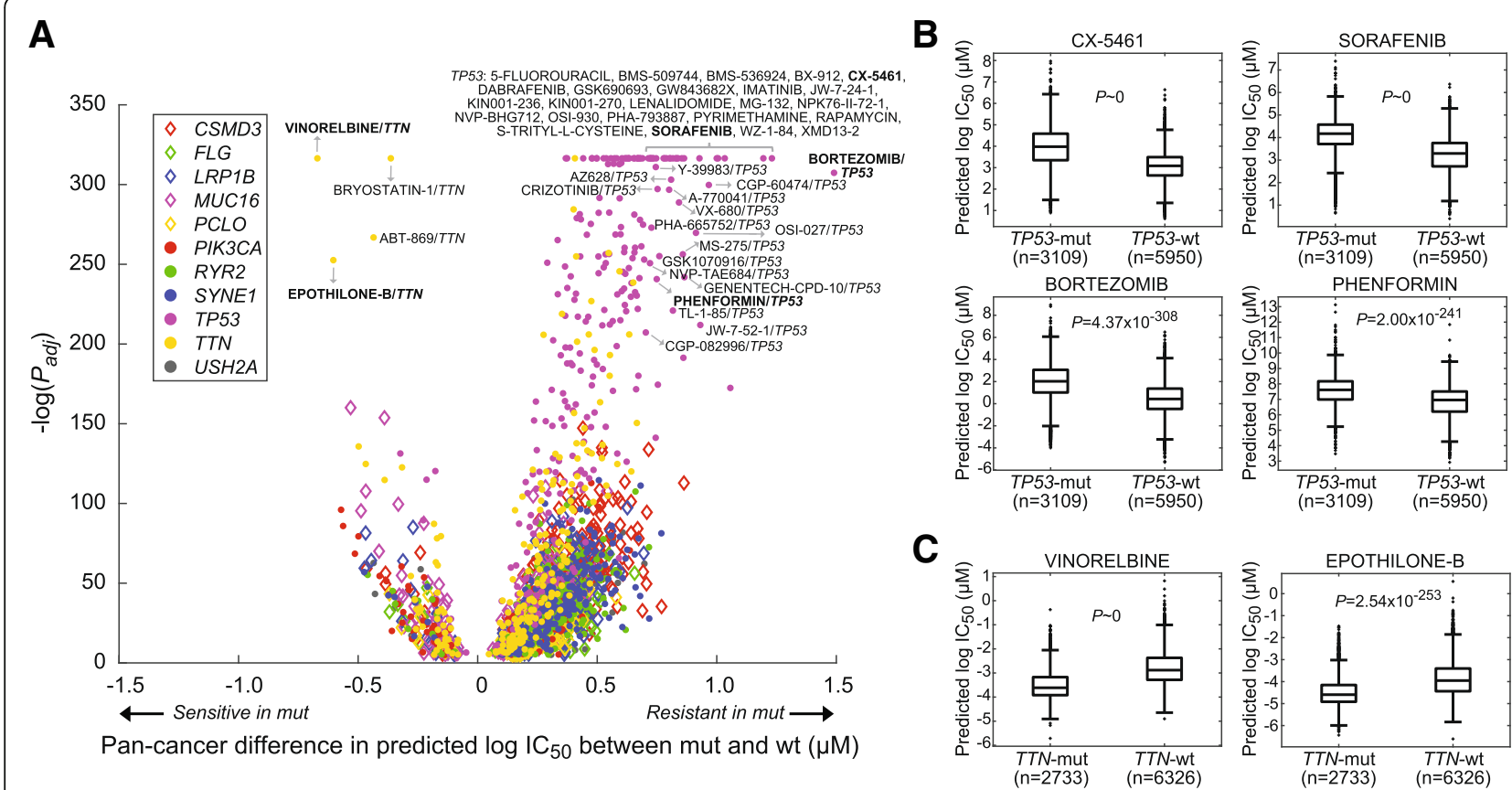

\footnotetext{
Fig. 4 Associations of gene mutations to predicted drug response in TCGA - pan-cancer study. (a) Gene mutations significantly associated with predicted drug response across all TCGA samples. Here only the 11 genes with mutation rates larger than $10 \%$ were analyzed. Nodes labeled with names are those with extreme significance (adjust $P<1.0 \times 10^{-200}$ ) and magnitude of $\Delta \mathrm{I} C_{50}\left(\Delta \mathrm{C}_{50} \geq 0.7\right.$ or $\Delta \mathrm{I} \mathrm{C}_{50}<0$ ). (b, $\left.\mathbf{c}\right)$ Examples of drugs modulated by TP53 and TTN mutations, respectively
} 


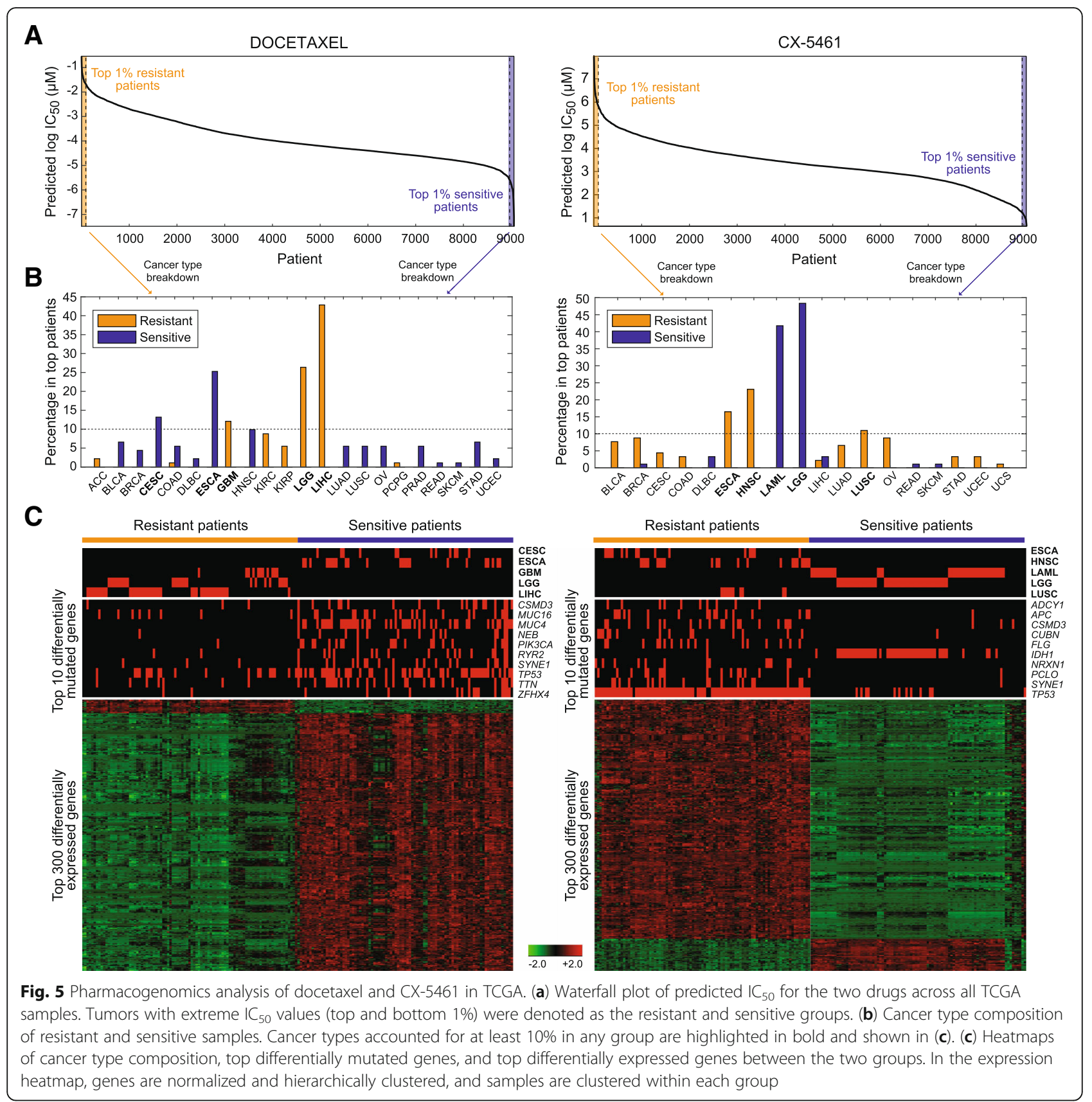

sensitive patients (Fig. 5c, left panel). We performed functional annotation analysis of the top 300 genes in Gene Ontology terms of biological processes and molecular functions using the Database for Annotation, Visualization and Integrated Discovery (DAVID) v6.7 $[33,34]$. While we did not observe any cluster of functions related to microtubule, through which docetaxel physically binds to the cell and regulate the cell cycle [35], these drug sensitivity-related genes were indeed predominantly enriched in functions governing the mitotic cell cycle (Table 4). The observation largely reflected the nature of the chemotherapeutic agent to target highly proliferative cells and the dependence of drug response on the ability to pass cell-cycle checkpoints. In addition to docetaxel, we analyzed a novel anti-cancer agent, CX-5461. This inhibitor of ribosomal RNA synthesis has been shown with anti-cancer properties in cancer cells $[36,37]$ and is now under phase I/II clinical trial in solid tumors (NCT number, NCT02719977). In hematopoietic malignancies, it was recently shown to outperform standard chemotherapy regimen in treating aggressive acute myeloid leukemia (LAML) [29], and its anti-cancer effects were dependent on wild-type TP53 [28, 29]. Concordantly, in our data, 
Table 4 Top GO clusters enriched in top 300 differentially expressed genes associated with predicted response to docetaxel

\begin{tabular}{|c|c|c|c|}
\hline GO ID & GO term & Num. genes & $P$-value \\
\hline \multicolumn{4}{|c|}{ Cluster 1 (enrichment score: 10.89) } \\
\hline GO:0007049 & cell cycle & 40 & $1.13 \times 10^{-10}$ \\
\hline GO:0022402 & cell cycle process & 33 & $3.51 \times 10^{-10}$ \\
\hline GO:0000279 & M phase & 32 & $1.01 \times 10^{-15}$ \\
\hline \multicolumn{4}{|c|}{ Cluster 2 (enrichment score: 3.96 ) } \\
\hline GO:0000166 & nucleotide binding & 56 & $1.95 \times 10^{-4}$ \\
\hline GO:0032553 & ribonucleotide binding & 54 & $2.74 \times 10^{-6}$ \\
\hline GO:0032555 & purine ribonucleotide binding & 54 & $2.74 \times 10^{-6}$ \\
\hline \multicolumn{4}{|c|}{ Cluster 3 (enrichment score: 3.45 ) } \\
\hline GO:0000278 & mitotic cell cycle & 26 & $1.01 \times 10^{-9}$ \\
\hline GO:0051726 & regulation of cell cycle & 15 & $8.48 \times 10^{-4}$ \\
\hline GO:0007346 & regulation of mitotic cell cycle & 12 & $3.09 \times 10^{-5}$ \\
\hline \multicolumn{4}{|c|}{ Cluster 4 (enrichment score: 2.47 ) } \\
\hline GO:0051327 & M phase of meiotic cell cycle & 8 & $9.46 \times 10^{-4}$ \\
\hline GO:0007126 & meiosis & 8 & $9.46 \times 10^{-4}$ \\
\hline GO:0051321 & meiotic cell cycle & 8 & $1.07 \times 10^{-3}$ \\
\hline \multicolumn{4}{|c|}{ Cluster 5 (enrichment score: 2.07) } \\
\hline GO:0051276 & chromosome organization & 13 & $8.64 \times 10^{-2}$ \\
\hline GO:0007059 & chromosome segregation & 6 & $9.34 \times 10^{-3}$ \\
\hline GO:0000070 & mitotic sister chromatid segregation & 5 & $2.45 \times 10^{-3}$ \\
\hline
\end{tabular}

Each cluster is represented by the largest three GO terms

Table 5 Top GO clusters enriched in top 300 differentially expressed genes associated with predicted response to CX-5461

\begin{tabular}{|c|c|c|c|}
\hline GO ID & GO term & Num. genes & $P$-value \\
\hline \multicolumn{4}{|c|}{ Cluster 1 (enrichment score: 8.65) } \\
\hline GO:0043062 & extracellular structure organization & 17 & $2.93 \times 10^{-9}$ \\
\hline GO:0030198 & extracellular matrix organization & 15 & $4.55 \times 10^{-10}$ \\
\hline GO:0005201 & extracellular matrix structural constituent & 13 & $2.64 \times 10^{-9}$ \\
\hline \multicolumn{4}{|c|}{ Cluster 2 (enrichment score: 6.13) } \\
\hline GO:0008544 & epidermis development & 18 & $2.35 \times 10^{-9}$ \\
\hline GO:0007398 & ectoderm development & 18 & $7.71 \times 10^{-9}$ \\
\hline GO:0030855 & epithelial cell differentiation & 8 & $4.60 \times 10^{-3}$ \\
\hline \multicolumn{4}{|c|}{ Cluster 3 (enrichment score: 4.23) } \\
\hline GO:0030199 & collagen fibril organization & 9 & $7.34 \times 10^{-9}$ \\
\hline GO:0032963 & collagen metabolic process & 6 & $5.37 \times 10^{-5}$ \\
\hline GO:0044259 & multicellular organismal macromolecule metabolic process & 6 & $8.96 \times 10^{-5}$ \\
\hline \multicolumn{4}{|c|}{ Cluster 4 (enrichment score: 2.84) } \\
\hline GO:0006928 & cell motion & 18 & $8.22 \times 10^{-4}$ \\
\hline GO:0016477 & cell migration & 13 & $9.51 \times 10^{-4}$ \\
\hline GO:0048870 & cell motility & 13 & $2.33 \times 10^{-3}$ \\
\hline \multicolumn{4}{|c|}{ Cluster 5 (enrichment score: 2.60 ) } \\
\hline GO:0060429 & epithelium development & 12 & $6.39 \times 10^{-4}$ \\
\hline GO:0030855 & epithelial cell differentiation & 8 & $4.60 \times 10^{-3}$ \\
\hline GO:0009913 & epidermal cell differentiation & 6 & $4.49 \times 10^{-3}$ \\
\hline
\end{tabular}


LAML and lymphoid neoplasm diffuse large B-cell lymphoma (DLBC) jointly accounted for $45.1 \%$ (41.8 and $3.3 \%$ ) of patients predicted be respond extremely well to CX-5461 (Fig. 5a-b, right panels). Of note, LGG comprised another $48.4 \%$ of the sensitive tumors (Fig. 5b, right panel). Nine of the top 10 differentially mutated genes were enriched in the resistant group and led by TP53 mutations (mutation rate, $95.6 \%$ in resistant vs. $13.2 \%$ in sensitive patients; Fig. 5c, right panel), echoing data from our pan-cancer analysis (Fig. 4a-b) and previous in vitro and in vivo investigations [28, 29]. IDH1 was the only gene preferentially mutated in sensitive tumors and largely marked LGG (mutated in 42 of 44 sensitive LGG; Fig. 5C, right panel). DAVID analysis of the top 300 differentially expressed genes highlighted differential mechanisms between solid and non-solid tumors, such as extracellular matrix and cell motion (Table 5). Altogether, the pharmacogenomics analyses revealed well-known resistance mechanisms of docetaxel and shed light on the potential of CX-5461 on hematopoietic malignancies and LGG.

\section{Discussion}

DNN is unquestionably one of the largest computational breakthroughs in the era of big data. Although promising results of our and other studies have demonstrated its ability of solving challenging bioinformatic tasks, the method has several fundamental limitations. For instance, due to high representational power and model complexity, the method suffers from overfitting and the requirement of large training data. Addressing this, the present study adopts a training-validation partition of training data to allow early stopping to the training process [38]. Future work may further incorporate dropout and regularization to DNNs. Also, by taking advantage of the transferability of neural networks, we used the huge volume of TCGA data to equip our model the ability of capturing representations of mutation and expression data. Transferring the learned parameters to initialize our model virtually increased the sample size of our training data. Our data from 100 iterations of model training suggest the stability of performance and insensitivity to the selection of training samples. With the availability of more large-scale drug screening data, we expect the proposed model to make even more accurate predictions and unveil subtle pharmacogenomics features. Furthermore, DeepDR may incorporate additional genomic mutation information, such as copy number alterations, into data matrices $M^{T C G A}$ and $M^{C C L E}$, to enrich the complexity of tumor mutation for model training and further reduce the training MSE. Because of the nature of DNNs as black boxes, the interpretability of results is typically limited. In this study, by integrating genomics profiles to the predictions, we systematically investigated how single gene mutations, as well as the interplay between cancer type, mutations, and biological functions, were associated with the predicted drug response. Several novel methods were recently proposed to extract features learned by neural networks, such as network-centric approach [39] and decomposition of predicted outputs by backpropagation onto specific input features [40] (reviewed in [41]). Future works may incorporate these methods to provide a landscape of pharmacogenomics and further reveal novel oncogenic genomics profiles.

\section{Conclusions}

This study addresses the need for a translation of pharmacogenomics features identified from pre-clinical cell line models to predict drug response of tumors. We developed a DNN model capable of extracting representative features of mutations and gene expression, and bridging knowledge learned from cancer cell lines and applications to tumors. We showed the reliability of the model and its superior performance than four different methods. Applying our model to the TCGA collection of tumors, we identified both well-studied and novel resistance mechanisms and drug targets. Overall, the proposed model is widely applicable to incorporate other omics data and to study a wider range of drugs, paving the way to the realization of precision oncology.

\section{Abbreviations}

ACC: adrenocortical cancer; BLCA: bladder urothelial carcinoma; BRCA: breast invasive carcinoma; CCLE: Cancer Cell Line Encyclopedia; CESC: cervical and endocervical cancer; $\mathrm{CHOL}$ : cholangiocarcinoma; COAD: colon adenocarcinoma; DL: deep learning; DLBC: diffuse large B-cell lymphoma; DNN: deep neural network; $E_{\text {enc: }}$ expression encoder; ER: estrogen receptor; ESCA: esophageal carcinoma; GBM: glioblastoma multiforme; HNSC: head and neck squamous cell carcinoma; $\mathrm{I}_{50}$ : half maximal inhibitory concentration; KICH: kidney chromophobe; KIRC: kidney clear cell carcinoma; KIRP: kidney papillary cell carcinoma; LAML: acute myeloid leukemia; LGG: lower grade glioma; LIHC: liver hepatocellular carcinoma; LUAD: lung adenocarcinoma; LUSC: lung squamous cell carcinoma; $M_{\text {enc: }}$ mutation encoder; MESO: mesothelioma; MSE: mean squared error; MUT: mutated; NSCLC: non-small cell lung cancer; Num: number; OV: ovarian serous cystadenocarcinoma; P: prediction network; P: P-value; PCA: principal component analysis; PCPG: pheochromocytoma and paraganglioma; PRAD: prostate adenocarcinoma; Rand Init: random initialization; READ: Rectum adenocarcinoma; SARC: Sarcoma; SKCM: Skin cutaneous melanoma; STAD: Stomach adenocarcinoma; SVM: Support vector machine; TCGA: The Cancer Genome Atlas; TGCT: Testicular germ cell tumor; THCA: Thyroid carcinoma; THYM: Thymoma; UCEC: Uterine corpus endometrioid carcinoma; UCS: Uterine carcinosarcoma; UVM: Uveal melanoma; WT: Wildtype

\section{Acknowledgements}

The authors greatly appreciate the brilliant and constructive inputs from reviewers and participants of the International Conference on Intelligent Biology and Medicine (ICIBM 2018).

\section{Availability of data and material}

The dataset supporting the conclusions of this article is included within the article. 


\section{Funding}

This research and this article's publication costs were supported partially by the NCl Cancer Center Shared Resources (NIH-NCI P30CA54174 to YC), NIH (CTSA 1UL1RR025767-01 to YC, and R01GM113245 to YH), CPRIT (RP160732 to YC), and San Antonio Life Science Institute (SALSI Innovation Challenge Award 2016 to YH and YC and SALSI Postdoctoral Research Fellowship to YCC). The funding sources had no role in the design of the study and collection, analysis, and interpretation of data and in writing the manuscript.

\section{About this supplement}

This article has been published as part of BMC Medical Genomics Volume 12 Supplement 1, 2019: Selected articles from the International Conference on Intelligent Biology and Medicine (ICIBM) 2018: medical genomics. The full contents of the supplement are available online at https:// bmcmedgenomics.biomedcentral.com/articles/supplements/volume-12 supplement-1.

\section{Authors' contributions}

All of the authors conceived the study. YCC, YH, and YC designed the model. YCC performed data analysis. YCC, YH, and YC interpreted the data. YCC, YH, and $Y C$ wrote the manuscript. All of the authors have read and approved the final manuscript.

\section{Ethics approval and consent to participate} Not applicable.

\section{Consent for publication}

Not applicable.

\section{Competing interests}

The authors declare that they have no competing interests.

\section{Publisher's Note}

Springer Nature remains neutral with regard to jurisdictional claims in published maps and institutional affiliations.

\section{Author details}

'Greehey Children's Cancer Research Institute, University of Texas Health Science Center at San Antonio, San Antonio, TX 78229, USA. ${ }^{2}$ Department of Electrical and Computer Engineering, The University of Texas at San Antonio, San Antonio, TX 78249, USA. ${ }^{3}$ Laboratory of Information Fusion Technology of Ministry of Education, School of Automation, Northwestern Polytechnical University, Xi'an 710072, Shaanxi, China. ${ }^{4}$ Department of Epidemiology and Biostatistics, University of Texas Health Science Center at San Antonio, San Antonio, TX 78229, USA

\section{Published: 31 January 2019}

\section{References}

1. Hanahan D, Weinberg RA. Hallmarks of cancer: the next generation. Cell. 2011;144(5):646-74.

2. Schmitt MW, Loeb LA, Salk JJ. The influence of subclonal resistance mutations on targeted cancer therapy. Nat Rev Clin Oncol. 2016;13(6):335-47.

3. Phillips KA, Veenstra DL, Oren E, Lee JK, Sadee W. Potential role of pharmacogenomics in reducing adverse drug reactions: a systematic review. JAMA : the journal of the American Medical Association. 2001 286(18):2270-9.

4. Hertz DL, Rae J. Pharmacogenetics of cancer drugs. Annu Rev Med. 2015;66:65-81

5. Mina M, Raynaud F, Tavernari D, Battistello E, Sungalee S, Saghafinia S, Laessle T, Sanchez-Vega F, Schultz N, Oricchio E, et al. Conditional selection of genomic alterations dictates Cancer evolution and oncogenic dependencies. Cancer Cell. 2017:32(2):155-68 e156

6. Park S, Lehner B. Cancer type-dependent genetic interactions between cancer driver alterations indicate plasticity of epistasis across cell types. Mol Syst Biol. 2015;11(7):824.

7. Iorio F, Knijnenburg TA, Vis DJ, Bignell GR, Menden MP, Schubert M, Aben N, Goncalves E, Barthorpe S, Lightfoot H, et al. A landscape of Pharmacogenomic interactions in Cancer. Cell. 2016;166(3):740-54.

8. Yang W, Soares J, Greninger P, Edelman EJ, Lightfoot H, Forbes S, Bindal N, Beare D, Smith JA, Thompson IR, et al. Genomics of drug sensitivity in
Cancer (GDSC): a resource for therapeutic biomarker discovery in cancer cells. Nucleic Acids Res. 2013:41(Database issue):D955-61.

9. Alipanahi B, Delong A, Weirauch MT, Frey BJ. Predicting the sequence specificities of DNA- and RNA-binding proteins by deep learning. Nat Biotechnol. 2015;33(8):831-8.

10. Zhou J, Troyanskaya OG. Predicting effects of noncoding variants with deep learning-based sequence model. Nat Methods. 2015;12(10):931-4.

11. Lin C, Jain S, Kim H, Bar-Joseph Z. Using neural networks for reducing the dimensions of single-cell RNA-Seq data. Nucleic Acids Res. 2017;45(17):e156.

12. Angermueller C, Lee HJ, Reik W, Stegle O. DeepCpG: accurate prediction of single-cell DNA methylation states using deep learning. Genome Biol. 2017; 18(1):67.

13. Chaudhary K, Poirion OB, Lu L, Garmire LX. Deep learning-based multiomics integration robustly predicts survival in liver Cancer. Clinical cancer research: an official journal of the American Association for Cancer Research. 2018;24(6):1248-59.

14. Wen M, Zhang Z, Niu S, Sha H, Yang R, Yun Y, Lu H. Deep-learning-based drug-target interaction prediction. J Proteome Res. 2017;16(4):1401-9.

15. Kadurin A, Aliper A, Kazennov A, Mamoshina P, Vanhaelen Q, Khrabrov K, Zhavoronkov A. The cornucopia of meaningful leads: applying deep adversarial autoencoders for new molecule development in oncology. Oncotarget. 2017;8(7):10883-90.

16. Preuer K, Lewis RPI, Hochreiter S, Bender A, Bulusu KC, Klambauer G. DeepSynergy: predicting anti-cancer drug synergy with deep learning Bioinformatics. 2018;34(9):1538-46.

17. Patro R, Duggal G, Love MI, Irizarry RA, Kingsford C. Salmon provides fast and bias-aware quantification of transcript expression. Nat Methods. 2017; 14(4):417-9.

18. Newton $Y$, Novak AM, Swatloski T, McColl DC, Chopra S, Graim K, Weinstein AS, Baertsch R, Salama SR, Ellrott K, et al. TumorMap: exploring the molecular similarities of Cancer samples in an interactive portal. Cancer Res. 2017:77(21):e111-4.

19. Barretina J, Caponigro G, Stransky N, Venkatesan K, Margolin AA, Kim S, Wilson CJ, Lehar J, Kryukov GV, Sonkin D, et al. The Cancer cell line encyclopedia enables predictive modelling of anticancer drug sensitivity. Nature. 2012:483(7391):603-7.

20. Cancer Cell Line Encyclopedia C. Genomics of drug sensitivity in Cancer C: Pharmacogenomic agreement between two cancer cell line data sets. Nature. 2015;528(7580):84-7.

21. Kowarik A, Templ M. Imputation with the R Package VIM. Journal of Statistical Software. 2016;74(7):1-16.

22. Alfons A, Templ M. Estimation of Social Exclusion Indicators from Complex Surveys. The R Package laeken 2013. 2013;54(15):25.

23. He K, Zhang X, Ren S, Sun J. Delving deep into rectifiers: surpassing humanlevel performance on imagenet classification. Proceedings of the IEEE international conference on computer vision. 2015;2015:1026-34.

24. Pumperla M. Keras + Hyperopt: A very simple wrapper for convenient hyperparameter optimization. 2016

25. Shetzer $Y$, Solomon H, Koifman G, Molchadsky A, Horesh S, Rotter V. The paradigm of mutant p53-expressing cancer stem cells and drug resistance. Carcinogenesis. 2014:35(6):1196-208.

26. Hientz K, Mohr A, Bhakta-Guha D, Efferth T. The role of p53 in cancer drug resistance and targeted chemotherapy. Oncotarget. 2017:8(5):8921-46.

27. Wang JB, Dong DF, Wang MD, Gao K. IDH1 overexpression induced chemotherapy resistance and IDH1 mutation enhanced chemotherapy sensitivity in glioma cells in vitro and in vivo. Asian Pac J Cancer Prev. 2014; 15(1):427-32.

28. Bywater MJ, Poortinga G, Sanij E, Hein N, Peck A, Cullinane C, Wall M, Cluse L, Drygin D, Anderes $\mathrm{K}$, et al. Inhibition of RNA polymerase I as a therapeutic strategy to promote cancer-specific activation of p53. Cancer Cell. 2012;22(1):51-65.

29. Hein N, Cameron DP, Hannan KM, Nguyen NN, Fong CY, Sornkom J, Wall M, Pavy M, Cullinane C, Diesch J, et al. Inhibition of pol I transcription treats murine and human AML by targeting the leukemia-initiating cell population. Blood. 2017;129(21):2882-95.

30. Wei JC, Meng FD, Qu K, Wang ZX, Wu QF, Zhang LQ, Pang Q, Liu C. Sorafenib inhibits proliferation and invasion of human hepatocellular carcinoma cells via up-regulation of p53 and suppressing FoxM1. Acta Pharmacol Sin. 2015:36(2):241-51.

31. Ling X, Calinski D, Chanan-Khan AA, Zhou M, Li F. Cancer cell sensitivity to bortezomib is associated with survivin expression and p53 status but not cancer cell types. J Exp Clin Cancer Res. 2010;29:8. 
32. Li P, Zhao M, Parris AB, Feng $X$, Yang $X$. p53 is required for metformininduced growth inhibition, senescence and apoptosis in breast cancer cells. Biochem Biophys Res Commun. 2015;464(4):1267-74.

33. Huang d W, Sherman BT, Lempicki RA. Systematic and integrative analysis of large gene lists using DAVID bioinformatics resources. Nat Protoc. 2009:4(1):44-57.

34. Huang d W, Sherman BT, Lempicki RA. Bioinformatics enrichment tools: paths toward the comprehensive functional analysis of large gene lists. Nucleic Acids Res. 2009:37(1):1-13.

35. Fulton B, Spencer CM. Docetaxel. A review of its pharmacodynamic and pharmacokinetic properties and therapeutic efficacy in the management of metastatic breast cancer. Drugs. 1996;51(6):1075-92.

36. Drygin D, Lin A, Bliesath J, Ho CB, O'Brien SE, Proffitt C, Omori M, Haddach M, Schwaebe MK, Siddiqui-Jain A, et al. Targeting RNA polymerase I with an oral small molecule CX-5461 inhibits ribosomal RNA synthesis and solid tumor growth. Cancer Res. 2011;71(4):1418-30

37. Xu H, Di Antonio M, McKinney S, Mathew V, Ho B, O'Neil NJ, Santos ND, Silvester J, Wei V, Garcia J, et al. CX-5461 is a DNA G-quadruplex stabilizer with selective lethality in BRCA1/2 deficient tumours. Nat Commun. 2017:8:14432

38. Angermueller $C$, Parnamaa T, Parts L, Stegle O. Deep learning for computational biology. Mol Syst Biol. 2016;12(7):878.

39. Yosinski J, Clune J, Nguyen A, Fuchs T, Lipson H. Understanding neural networks through deep visualization. In: arXiv preprint arXiv:150606579; 2015

40. Shrikumar A, Greenside P, Kundaje A. Learning important features through propagating activation differences. In: arXiv preprint arXiv:170402685; 2017.

41. Kalinin AA, Higgins GA, Reamaroon N, Soroushmehr S, Allyn-Feuer A, Dinov ID, Najarian K, Athey BD. Deep learning in pharmacogenomics: from gene regulation to patient stratification. In: arXiv preprint arXiv:180108570; 2018.

Ready to submit your research? Choose BMC and benefit from:

- fast, convenient online submission

- thorough peer review by experienced researchers in your field

- rapid publication on acceptance

- support for research data, including large and complex data types

- gold Open Access which fosters wider collaboration and increased citations

- maximum visibility for your research: over $100 \mathrm{M}$ website views per year

At $\mathrm{BMC}$, research is always in progress.

Learn more biomedcentral.com/submissions 